\title{
USING DELTA RANGE ON GPS POSITIONING, FAULT DETECTION AND FAULT EXCLUSION
}

\author{
Yi-Hsueh Tsai ${ }^{*}$ Fan-Ren Chang ${ }^{*}$, and Wen-Chieh Yang ${ }^{* *}$ \\ ${ }^{*}$ Dept. of Electrical Engineering, National Taiwan University, Taipei, Taiwan \\ ${ }^{* *}$ Div. of Research and Development, Intelligent Business Technology Inc., Taipei, Taiwan
}

\begin{abstract}
Conventionally, the well known position-velocity-acceleration (PVA) model is adopted as the dynamic model of Kalman filter for GPS positioning. However, as a moving vehicle corners at faster speeds, the conventional PVA model without using extra sensors can no longer be adequate for describing the motion of the vehicle. Thus, the positioning result of the vehicle will be less accurate, and normalized innovation squared (NIS) is not suitable as the test statistic for FDE anymore. Therefore, the delta range (DR) equation is proposed to accurately model the dynamic behavior of a maneuvering vehicle. Simulation results show that the proposed DR can perform both positioning and FDE well as the vehicle manoeuvres. Copyright $\mathbb{C} 2005$ IFAC
\end{abstract}

Keywords: Global positioning systems, Kalman filter, Position estimation, Fault detection, Fault isolation, and Threshold value

\section{INTRODUCTION}

A GPS modernization policy has been approved by US government to improve the performance of the current navigation system. In the near future, the Block IIR-M GPS satellite carrying L2 signal will be launched. The main advantages of the modernized GPS receiver are performance enhancement in both positioning accuracy, and fault detection and exclusion (FDE). FDE is a crucial issue, as satellite failures may result in serious deviations of aircrafts from their intended flight paths. Sometimes the term "receiver autonomous integrity monitoring (RAIM)" is adopted for the similar concern. The purpose of FDE is to "detect" the presence of unacceptably large position error and, further, to "exclude" the source causing the error. To achieve this goal, a number of useful FDE algorithms have been published over the last few decades. Brown and Hwang (1986) proposed a dynamic algorithm by using a parallel bank of Kalman filters to detect failures. The GPS measurement is first subtracted by a specified type of failure and then applied to a Kalman filter. For each type of failure to be protected, a corresponding Kalman filter is necessary. $\mathrm{Da}$ and Lin (1995) proposed a dynamic algorithm to detect GPS failure by using two Kalman filters. One processes only measurement from a GPS receiver, and the other processes measurements from the GPS receiver and some inertial navigation sensors (INS). By using the estimated states and their covariance matrices produced from these two Kalman filters, a test statistic can be obtained for performing GPS satellite fault detection. The proposed method, however, only focuses on fault detection. Young and McGraw (2003) also proposed a dynamic algorithm to perform FDE by using two Kalman filters. Measurements from INS sensors are also needed in this algorithm. Moreover, a parallel bank of Kalman filters is adopted to exclude the failed satellite. It can be achieved by removing one of the visible satellites at a time for each Kalman filter in the parallel bank.

Conventionally, the well known PVA model is adopted as the dynamic model of Kalman filter for GPS positioning. Under the hypothesis that no failure is taking place and the vehicle is moving with a constant velocity, the normalized innovation squared of Kalman filter will be chi-square distributed and thus can be used as the test statistic for fault detection and exclusion (FDE). However, as a moving vehicle accelerates or slows down more rapidly, or as the vehicle corners at faster speeds, the conventional PVA model without using extra sensors (such as INS) can no 
longer be adequate for describing the motion of the vehicle. Therefore, the positioning result of the vehicle becomes less accurate, and NIS will not be chi-square distributed and is not suitable as the test statistic for FDE anymore. Thus, the delta range (DR) equation is proposed by the authors to accurately model the dynamic behavior of a maneuvering vehicle. Two simulations are conducted to verify the proposed algorithms. First, Monte Carlo simulations are executed to compare the proposed DR equation with the conventional PVA model, where the vehicle is assumed to be moving with a constant velocity. Simulation results show that, in comparison with the PVA model, the DR demonstrates higher performance in detecting and excluding small failures. However, in detecting and excluding large failures, PVA model demonstrates higher performance than DR. In the second simulation, it is assumed that the vehicle will first complete a half circle at a specified time and then slows down rapidly at another time. Simulation results show that, in comparison with the PVA model, the proposed DR can provide more accurate positioning result and NIS. In summary, in comparison with the PVA model, the proposed DR can perform both positioning and FDE well as the vehicle manoeuvres.

\section{LINEARIZATION OF THE IONO- SPHERE-FREE MEASUREMENTS FOR KALMAN FILTER}

According to (Hofmann-Wellenhof, et al., 1997) and (Fontana, et al., 2001), the modernized GPS receiver can provide three different types of ionosphere-free measurements: the pseudorange measurement $\boldsymbol{\rho}(k)$, the delta range measurement $\phi(k)$, and the Doppler shift measurement $\psi(k)$. Assume that the nominal vector, comprised of the nominal user position plus the nominal user clock draft, is set as the reference point $\mathbf{x}_{r e f}(k)$ with dimension $4 \times 1$. According to (Hofmann-Wellenhof, et al., 1997), the linearization of $\boldsymbol{\rho}(k), \phi(k)$ and $\boldsymbol{\psi}(k)$ can be obtained as

$$
\begin{aligned}
\boldsymbol{\rho}(k)= & \mathbf{d}(k)+\mathbf{H}(k)\left[\mathbf{x}(k)-\mathbf{x}_{r e f}(k)\right]+\mathbf{w}(k), \\
\phi(k)= & \mathbf{d}(k)-\mathbf{d}(k-1)+\mathbf{H}(k)\left[\mathbf{x}(k)-\mathbf{x}_{r e f}(k)\right] \\
& -\mathbf{H}(k-1)\left[\mathbf{x}(k-1)-\mathbf{x}_{r e f}(k-1)\right]+\boldsymbol{\omega}(k),
\end{aligned}
$$

and

$$
\boldsymbol{\psi}(k)=\dot{\mathbf{d}}(k)+\mathbf{H}(k) \mathbf{x}_{v}(k)+\mathbf{w}_{v}(k),
$$

where $\mathbf{d}(k)$ is the $n \times 1$ predicted range vector based on the reference point $\mathbf{x}_{r e f}(k) ; \dot{\mathbf{d}}(k)$ is the $n \times 1$ predicted range rate vector based on the reference point $\mathbf{x}_{\text {ref }}(k)$; $\mathbf{H}(k)$ is the $n \times 4$ observation matrix arrived at by linearizing around $\mathbf{x}_{r e f}(k) ; \mathbf{x}(k)$ is the $4 \times 1$ state vector comprised of the true position, plus the user clock draft; $\mathbf{x}_{v}(k)$ is the $4 \times 1$ state vector comprised of the true velocity, plus the user clock draft rate; $\mathbf{w}(k)$ is the $n \times 1$ pseudorange measurement noises, and is assumed to be zero mean white Gaussian with the covariance matrices $\mathbf{R}(k)$; $\boldsymbol{\omega}(k)$ is the $n \times 1$ delta range measurement noises, and is assumed to be zero mean white Gaussian with the covariance matrices $\boldsymbol{\Omega}(k)$; $\mathbf{w}_{v}(k)$ is the $n \times 1$ Doppler shift measurement noises, and is assumed to be zero mean white Gaussian with the $n \times n$ covariance matrices $\mathbf{R}_{v}(k) ; n$ is the number of visible satellites. Define $\mathbf{z}(k) \equiv \boldsymbol{\rho}(k)-\mathbf{d}(k)+\mathbf{H}(k) \mathbf{x}_{r e f}(k)$ and substitute $\mathbf{z}(k)$ into (1), and then the linearized pseudorange measurement equation becomes

$$
\mathbf{z}(k)=\mathbf{H}(k) \mathbf{x}(k)+\mathbf{w}(k) \text {. }
$$

In addition, define $\mathbf{u}(k)=\phi(k)-\mathbf{d}(k)+\mathbf{d}(k-1)+$ $\mathbf{H}(k) \mathbf{x}_{r e f}(k)-\mathbf{H}(k-1) \mathbf{x}_{r e f}(k-1)$ and substitute $\mathbf{u}(k)$ into (2), and then the linearized delta range measurement equation becomes

$$
\mathbf{u}(k)=\mathbf{H}(k) \mathbf{x}(k)-\mathbf{H}(k-1) \mathbf{x}(k-1)+\boldsymbol{\omega}(k) .
$$

Moreover, define $\mathbf{z}_{v}(k)=\psi(k)-\mathbf{d}(k)$ and substitute $\mathbf{z}_{v}(k)$ into (3), and then the linearized Doppler shift measurement becomes

$$
\mathbf{z}_{v}(k)=\mathbf{H}(k) \mathbf{x}_{v}(k)+\mathbf{w}_{v}(k) .
$$

\section{CONVENTIONAL ALGORITHMS: USING PVA MODEL ON KALMAN FILTER}

Conventionally, the well known position- velocity-acceleration (PVA) model is adopted as the dynamic model of Kalman filter for GPS positioning. As such, the dynamic and measurement equations can be obtained as

$$
\left\{\begin{array}{l}
\mathbf{x}_{P V A}(k)=\mathbf{\Phi}_{P V A} \mathbf{x}_{P V A}(k-1)+\mathbf{v}_{P V A}(k) \\
\mathbf{z}_{P V A}(k)=\mathbf{H}_{P V A}(k) \mathbf{x}_{P V A}(k)+\mathbf{w}_{P V A}(k)
\end{array},\right.
$$

where $\mathbf{z}_{P V A}(k)=\left[\mathbf{z}^{T}(k) \mathbf{z}_{v}^{T}(k)\right]^{T}$ is the $2 n \times 1$ measurement vector; $\mathbf{x}_{P V A}(k)=\left[\mathbf{x}^{T}(k) \mathbf{x}_{v}^{T}(k) \mathbf{x}_{a}^{T}(k)\right]^{T}$ is the $12 \times 1$ state vector and $\mathbf{x}_{a}(k)$ is the $4 \times 1$ state vector comprised of the true acceleration, plus the change rate of the user clock draft rate; $\boldsymbol{\Phi}_{P V A}=\left[\begin{array}{cccc}\mathbf{I}_{4} & t_{S} \mathbf{I}_{4} & \frac{1}{2} t_{S}^{2} \mathbf{I}_{4} \\ \mathbf{0} & \mathbf{I}_{4} & t_{S} \mathbf{I}_{4} \\ \mathbf{0} & \mathbf{0} & \mathbf{I}_{4}\end{array}\right]$ is the $12 \times 12$ transition matrix and $\mathbf{I}_{4}$ is a $4 \times 4$ identity matrix; $\mathbf{H}_{P V A}=\left[\begin{array}{ccc}\mathbf{H}(k) & \mathbf{0} & \mathbf{0} \\ \mathbf{0} & \mathbf{H}(k) & \mathbf{0}\end{array}\right]$ is the $2 n \times 12$ measurement matrix; $\mathbf{v}_{P V A}(k)$ is the $12 \times 1$ process noise, and is assumed to be zero mean white Gaussian with the covariance matrix $\mathbf{Q}_{P V A}=q_{P V A}\left[\begin{array}{ccc}\frac{1}{20} t_{S}^{5} \mathbf{I}_{4} & \frac{1}{8} t_{S}^{4} \mathbf{I}_{4} & \frac{1}{6} t_{S}^{3} \mathbf{I}_{4} \\ \frac{1}{8} t_{S}^{4} \mathbf{I}_{4} & \frac{1}{3} t_{S}^{3} \mathbf{I}_{4} & \frac{1}{2} t_{S}^{2} \mathbf{I}_{4} \\ \frac{1}{6} t_{S}^{3} \mathbf{I}_{4} & \frac{1}{2} t_{S}^{2} \mathbf{I}_{4} & t_{S} \mathbf{I}_{4}\end{array}\right]$; $\mathbf{w}_{P V A}(k)$ is the $2 n \times 1$ measurement noise, and is assumed to be zero mean white Gaussian with the covariance matrix $\mathbf{R}_{P V A}(k)=\operatorname{diag}\left\{\mathbf{R}(k), \mathbf{R}_{v}(k)\right\}$; furthermore, $\mathbf{w}_{P V A}(k)$ and $\mathbf{v}_{P V A}(k)$ are assumed to be mutually independent; and $t_{S}$ is the sampling time. According to (Bar-Shalom and Fortman, 1993), the $12 \times 1$ updated state estimate can be obtained as

$$
\hat{\mathbf{x}}_{P V A}(k \mid k)=\boldsymbol{\Phi}_{P V A} \hat{\mathbf{x}}_{P V A}(k-1 \mid k-1)+\mathbf{K}_{P V A}(k) \widetilde{\mathbf{z}}_{P V A}(k),(8)
$$

with the $12 \times 12$ updated state covariance matrix

$$
\mathbf{P}_{P V A}(k \mid k)=\left[\mathbf{P}_{P V A}^{-1}(k \mid k-1)+\mathbf{H}_{P V A}^{T}(k) \mathbf{R}_{P V A}(k) \mathbf{H}_{P V A}(k)\right]^{-1},
$$


where $\quad \mathbf{P}_{P V A}(k \mid k-1)=\boldsymbol{\Phi}_{P V A} \mathbf{P}_{P V A}(k-1 \mid k-1) \boldsymbol{\Phi}_{P V A}^{T}+\mathbf{Q}_{P V A}$ is the $12 \times 12$ state prediction covariance matrix, $\mathbf{K}_{P V A}(k)=\mathbf{P}_{P V A}(k \mid k) \mathbf{H}_{P V A}^{T}(k) \mathbf{R}_{P V A}^{-1}(k)$ is the $12 \times 2 n$ Kalman gain, and

$$
\widetilde{\mathbf{z}}_{P V A}(k)=\mathbf{z}_{P V A}(k)-\mathbf{H}_{P V A}(k) \boldsymbol{\Phi}_{P V A} \hat{\mathbf{x}}_{P V A}(k-1 \mid k-1)
$$

is the innovation vector with the covariance matrix

$$
\mathbf{S}_{P V A}(k)=\mathbf{H}_{P V A}(k) \mathbf{P}_{P V A}(k \mid k-1) \mathbf{H}_{P V A}^{T}(k)+\mathbf{R}_{P V A}(k) .
$$

In terms of the innovation and its covariance matrix, the normalized innovation squared (NIS) can be defined as

$$
S_{P V A}(k) \equiv \widetilde{\mathbf{z}}_{P V A}^{T}(k) \mathbf{S}_{P V A}^{-1}(k) \widetilde{\mathbf{z}}_{P V A}(k) .
$$

Under the hypothesis that no failure is taking place, the vehicle is moving with a constant velocity, and $\mathbf{Q}_{P V A}(k)$ is set as zero, the distribution of $s_{P V A}(k)$ is $\chi^{2}(2 n)$, where $\chi^{2}(v)$ represents the chi-square distribution with $v$ degrees of freedom and $n$ is the number of visible GPS satellites. Then $s_{P V A}(k)$ will be compared with a threshold $T_{d}$ to judge whether the system is failed or not. The threshold value under a specified false alarm rate (FAR) can be calculated directly through the cumulative distribution function of $\chi^{2}(2 n)$. The resulting threshold values under FAR equal to $1 / 15000$ (RTCA/DO-229, 1997) are listed in Table 1.

\begin{tabular}{ccc}
\multicolumn{3}{c}{ Table 1: detection thresholds for $\mathrm{S}_{P V A}(k)$} \\
$\begin{array}{c}\text { Number of } \\
\text { visible satellites }\end{array}$ & $\begin{array}{c}\text { degrees of } \\
\text { freedom }\end{array}$ & $\begin{array}{c}\text { Detection } \\
\text { threshold }\end{array}$ \\
\hline 4 & 8 & 32.8089 \\
5 & 10 & 36.5882 \\
6 & 12 & 40.1979 \\
7 & 14 & 43.6794 \\
8 & 16 & 47.0593 \\
9 & 18 & 50.3562 \\
\hline
\end{tabular}

After the detection of satellite malfunction, the pseudorange and the Doppler shift measurement associated to the failed satellite must be excluded to ensure uninterrupted navigation. According to (Young, 2003), the fault exclusion algorithm based on a parallel bank of Kalman filter is derived as follows. Solutions are formed by removing one of the visible satellites at a time. To be precise, the number of Kalman filters in the parallel bank is equal to the number of visible satellites. As for the $i$ th Kalman filter, the pseudorange and the Doppler shift measurement are obtained from all but the $i$ th visible satellites. Then a corresponding normalized innovation squared $s_{P V A, i}(k)$ can be obtained, and a satellite will be identified as failed if and only if the following equation is satisfied

$$
\left\{\begin{array}{l}
s_{P V A, i}(k) \leq T_{e}, i=n_{f}(k) \\
s_{P V A, i}(k)>T_{e}, \text { otherwise }
\end{array},\right.
$$

where $n_{f}(k)$ is the channel number of the failed satellite at time $k, T_{e}$ is a selected threshold value for satellite fault exclusion. Under the hypothesis that no failure is taking place and the vehicle is moving with a constant acceleration, the distribution of $s_{P V A, i}(k)$ will be $\chi^{2}(2 n-2)$. A specified FAR will be given for exclusion. The resulting threshold values under FAR equal to $1 / 250$ are listed in Table 2 .

\begin{tabular}{ccc}
\multicolumn{3}{c}{ Table 2: exclsuion thresholds for $\mathrm{S}_{P V A, i}(k)$} \\
$\begin{array}{c}\text { Number of } \\
\text { visible satellites }\end{array}$ & $\begin{array}{c}\text { degrees of } \\
\text { freedom }\end{array}$ & $\begin{array}{c}\text { Exclusion } \\
\text { threshold }\end{array}$ \\
\hline 4 & 6 & 20.7912 \\
5 & 8 & 24.3521 \\
6 & 10 & 27.7216 \\
7 & 12 & 30.9570 \\
8 & 14 & 34.0913 \\
9 & 16 & 37.1461 \\
\hline
\end{tabular}

According to (Parkinson and Axelrad, 1988), the user may perform integrity checking and failed satellite exclusion through the following five steps:

Step 1: Compute the normalized innovation squared, $S_{P V A}(k)$, using data of all visible satellites from (12).

Step 2: If $s_{P V A}(k)$ is larger than $T_{d}$, then declare detection of a failure, and goto Step 3. Otherwise, all satellites are assumed to operate properly, and the integrity check has been completed.

Step 3: Compute the normalized innovation squared, $s_{P V A, i}(k)$, using data from all but $i$ th visible satellites, for $i=1, \ldots, n$.

Step 4: If one of the $s_{P V A, i}(k)$ is less than the exclusion threshold, $T_{e}$, and all others are larger than $T_{e}$, identify the satellite omitted from the $i$ th Kalman filter as the failed one. If two or more of $s_{P V A, i}(k)$ are below the threshold, the failed satellite cannot be excluded. (see (13))

Step 5: If a failed satellite is detected and excluded, use the navigation solution formed by omitting the failed one. If a failed satellites is detected, but cannot be excluded, use the all-in-view solution if necessary, but recognize that the positioning accuracy is degraded.

When the PVA model is applied, the vehicle position becomes less accurate as a moving vehicle accelerates or slows down more rapidly, or as the vehicle corners at faster speeds. Under this situation, the distribution of NIS will not be unknown, and thus is not suitable to be the test statistic for FDE anymore. Therefore, the delta range (DR) equation is proposed to accurately model the dynamic behavior of a maneuvering vehicle.

\section{USING DELTA RANGE EQUATION ON KALMAN FILTER FOR A MANEUVERING VEHI- CLE}

Vehicle manoeuvres, referring to unpredictable changes in the vehicle, may cause serious inaccuracies in modeling the system. Therefore, the positioning result of the vehicle becomes less accurate, and NIS will not be chi-square distributed and thus is not suitable to be the test statistic for FDE. To overcome this problem, the delta range (DR) equation is adopted as the dynamic equation for the Kalman filter. According to (5), the delta range equation can be described as

$$
\mathbf{u}(k)=\mathbf{H}(k) \mathbf{x}(k)-\mathbf{H}(k-1) \mathbf{x}(k-1)+\boldsymbol{\omega}(k),
$$

where $\mathbf{x}(k)$ is the $4 \times 1$ state vector comprised of the true position, plus the user clock draft; $\omega(k)$ is assumed to be zero mean white Gaussian noise with covariance matrices $\Omega(k)$. Assume $\mathbf{L}(k)$ is a $4 \times n$ 
matrix satisfying $\mathbf{L}(k) \mathbf{H}(k)=\mathbf{I}_{4}$, where $\mathbf{I}_{4}$ is a $4 \times 4$ identity matrix. Multiply $\mathbf{L}(k)$ on both sides of (14), and then the following equation can be derived

$$
\mathbf{L}(k) \mathbf{u}(k)=\mathbf{x}(k)-\boldsymbol{\Phi}_{D R}(k) \mathbf{x}(k-1)-\mathbf{v}_{D R}(k),
$$

where $\boldsymbol{\Phi}_{D R}(k)=\mathbf{L}(k) \mathbf{H}(k-1)$ is the transition matrix, and $\mathbf{v}_{D R}(k)=-\mathbf{L}(k) \boldsymbol{\omega}(k)$ is the $4 \times 1$ noise vector, assumed to be zero mean white Gaussian with the covariance matrix $\mathbf{Q}_{D R}(k)=\mathbf{L}(k) \boldsymbol{\Omega}(k) \mathbf{L}^{T}(k)$. To give a simple form of $\mathbf{Q}_{D R}(k)$, we can select $\mathbf{L}(k)$ as

$$
\mathbf{L}(k)=\left(\mathbf{H}^{T}(k) \boldsymbol{\Omega}^{-1}(k) \mathbf{H}(k)\right)^{-1} \mathbf{H}^{T}(k) \mathbf{\Omega}^{-1}(k) .
$$

Then the covariance matrix $\mathbf{Q}_{D R}(k)$ can be derived as $\mathbf{Q}_{D R}(k)=\left(\mathbf{H}^{T}(k) \boldsymbol{\Omega}^{-1}(k) \mathbf{H}(k)\right)^{-1}$. As a result, $\mathbf{Q}_{D R}(k)$ will have a simpler form if $\mathbf{L}(k)$ is chosen as in (16).

By applying (4) and (15) to the Kalman filter, a moving vehicle can be described by the following dynamic and measurement equations

$$
\left\{\begin{array}{l}
\mathbf{x}(k)=\mathbf{\Phi}_{D R}(k) \mathbf{x}(k-1)+\mathbf{L}(k) \mathbf{u}(k)+\mathbf{v}_{D R}(k), \\
\mathbf{z}(k)=\mathbf{H}(k) \mathbf{x}(k)+\mathbf{w}(k)
\end{array}\right.
$$

where $\mathbf{x}(k)$ is the $4 \times 1$ state vector; $\boldsymbol{\Phi}_{D R}(k)$ is the $4 \times 4$ transition matrix; $\mathbf{z}(k)$ is the $n \times 1$ measurement vectors; $\mathbf{u}(k)$ is the $n \times 1$ input vectors; $\mathbf{L}(k)$ is the $4 \times n$ input gain matrix; $\mathbf{v}_{D R}(k)$ is the $4 \times 1$ process noise, and is assumed to be zero mean white Gaussian with the covariance matrices $\mathbf{Q}_{D R}(k) ; \mathbf{w}(k)$ is the $n \times 1$ measurement noise, and is assumed to be zero mean white Gaussian with the covariance matrices $\mathbf{R}(k)$; furthermore, $\mathbf{v}_{D R}(k)$ and $\mathbf{w}(k)$ are assumed to be mutually independent. According to (Bar-Shalom, 1993), the $4 \times 1$ updated state estimate can be obtained as

$$
\begin{aligned}
\hat{\mathbf{x}}_{D R}(k \mid k)= & \boldsymbol{\Phi}_{D R}(k) \hat{\mathbf{x}}_{D R}(k-1 \mid k-1) \\
& +\mathbf{L}(k) \mathbf{u}(k)+\mathbf{K}_{D R}(k) \widetilde{\mathbf{z}}(k)
\end{aligned}
$$

with the $4 \times 4$ updated state covariance matrix

$$
\mathbf{P}_{D R}(k \mid k)=\left[\mathbf{P}_{D R}^{-1}(k \mid k-1)+\mathbf{H}^{T}(k) \mathbf{R}(k) \mathbf{H}(k)\right]^{-1},
$$

where $\mathbf{P}_{D R}(k \mid k-1)=\boldsymbol{\Phi}_{D R}(k) \mathbf{P}_{D R}(k-1 \mid k-1) \boldsymbol{\Phi}_{D R}^{T}(k)$ $+\mathbf{Q}_{D R}(k)$ is the $4 \times 4$ state prediction covariance matrix; $\mathbf{K}_{D R}(k)=\mathbf{P}_{D R}(k \mid k) \mathbf{H}^{T}(k) \mathbf{R}^{-1}(k)$ is the $4 \times n$ Kalman Gain; and

$$
\begin{aligned}
& \widetilde{\mathbf{z}}_{D R}(k)=\mathbf{z}(k) \\
& \quad-\mathbf{H}(k)\left[\boldsymbol{\Phi}_{D R}(k) \hat{\mathbf{x}}_{D R}(k-1 \mid k-1)+\mathbf{L}(k) \mathbf{u}(k)\right]
\end{aligned}
$$

is the innovation vector with the covariance matrix

$$
\mathbf{S}_{D R}(k)=\mathbf{H}(k) \mathbf{P}_{D R}(k \mid k-1) \mathbf{H}^{T}(k)+\mathbf{R}(k) .
$$

In terms of the innovation and its covariance matrix, the normalized innovation squared (NIS) can be defined as

$$
s_{D R}(k) \equiv \widetilde{\mathbf{z}}_{D R}^{T}(k) \mathbf{S}_{D R}^{-1}(k) \widetilde{\mathbf{z}}_{D R}(k) .
$$

Under the hypothesis that no failure is taking place, the distribution of $s_{D R}(k)$ is $\chi^{2}(n)$. Then $s_{D R}(k)$ will be compared with a threshold $T_{d}$ to judge whether the system is failed or not. The threshold value under a specified FAR can be calculated directly through the cumulative distribution function of $\chi^{2}(n)$. The resulting threshold values under FAR equal to $1 / 15000$ (RTCA/DO-229, 1997) are listed in Table 3.

\begin{tabular}{ccc}
\multicolumn{3}{c}{ Table 3: detection thresholds for $\mathrm{S}_{D R}(k)$} \\
$\begin{array}{c}\text { Number of } \\
\text { visible satellites }\end{array}$ & $\begin{array}{c}\text { Degrees of } \\
\text { freedom }\end{array}$ & $\begin{array}{c}\text { Detection } \\
\text { threshold }\end{array}$ \\
\hline 4 & 4 & 24.3914 \\
5 & 5 & 26.6521 \\
6 & 6 & 28.7899 \\
7 & 7 & 30.8356 \\
8 & 8 & 32.8089 \\
9 & 9 & 34.7232 \\
\hline
\end{tabular}

After the detection of satellite malfunction, the pseudorange and the delta range measurement associated to the fault satellite must be excluded to ensure uninterrupted navigation. The fault exclusion algorithm based on a parallel bank of Kalman filter is derived as follows. The number of Kalman filters needed in this parallel bank is equal to the number of visible satellites. As for the $i$ th Kalman filter, the pseudorange and the delta range measurement are obtained from all but the $i$ th visible satellites. Then a corresponding normalized innovation squared $s_{D R, i}(k)$ can be obtained, and a satellite will be identified as failed if and only if the following equation is satisfied

$$
\left\{\begin{array}{l}
s_{D R, i}(k) \leq T_{e}, i=n_{f}(k) \\
s_{D R, i}(k)>T_{e}, \text { otherwise }
\end{array},\right.
$$

where $n_{f}(k)$ is the channel number of the failed satellite at time $k, T_{e}$ is a selected threshold value for satellite fault exclusion. Under the hypothesis that no failure is taking place, the distribution of $s_{D R, i}(k)$ is $\chi^{2}(n-1)$. The resulting threshold values under FAR equal to $1 / 250$ are listed in Table 4 . Fig. 1 depicts the system diagram for satellite fault detection and exclusion algorithm using DR equation.

\begin{tabular}{ccc}
\multicolumn{3}{c}{ Table 4: exclsuion thresholds for $\mathrm{S}_{D R, i}(k)$} \\
$\begin{array}{c}\text { Number of } \\
\text { visible satellites }\end{array}$ & $\begin{array}{c}\text { degrees of } \\
\text { freedom }\end{array}$ & $\begin{array}{c}\text { Exclusion } \\
\text { threshold }\end{array}$ \\
\hline 4 & 3 & 14.7955 \\
5 & 4 & 16.9238 \\
6 & 5 & 18.9074 \\
7 & 6 & 20.7912 \\
8 & 7 & 22.6007 \\
9 & 8 & 24.3521 \\
\hline
\end{tabular}

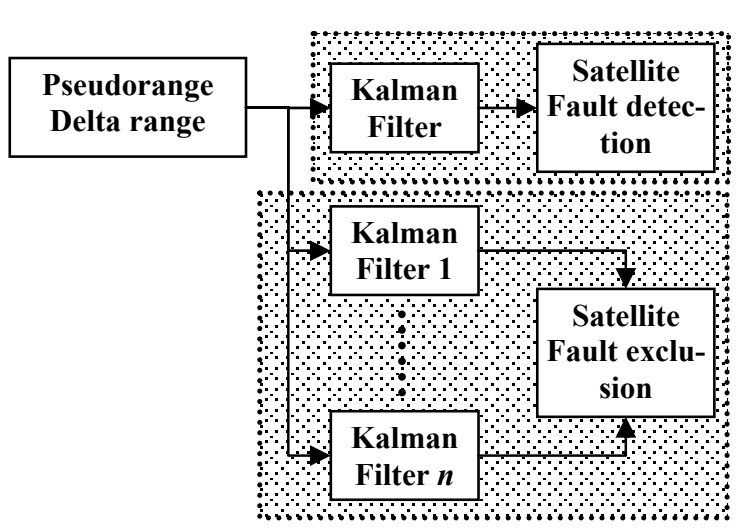

Fig. 1: system diagram for FDE using DR equation 


\section{SIMULATION RESULTS}

Two simulations are conducted to verify the proposed algorithms. First, Monte Carlo simulations are executed to compare the proposed DR equation with the conventional PVA model, where the vehicle is assumed to be moving with a constant velocity. In the second simulation, it is assumed that the vehicle will first complete a half circle at a specified time and then slows down rapidly at another time. The goal of the simulation is to verify that the proposed DR can still perform both positioning and FDE well as the vehicle manoeuvres. The software package "Satellite Navigation TOOLBOX for Matlab" by GPSoft LLC is adopted in the simulation. It assumed a 24-satellite constellation with perfectly circular orbits. In addition, the receiver mask angle is set as $7.5^{\circ}$.

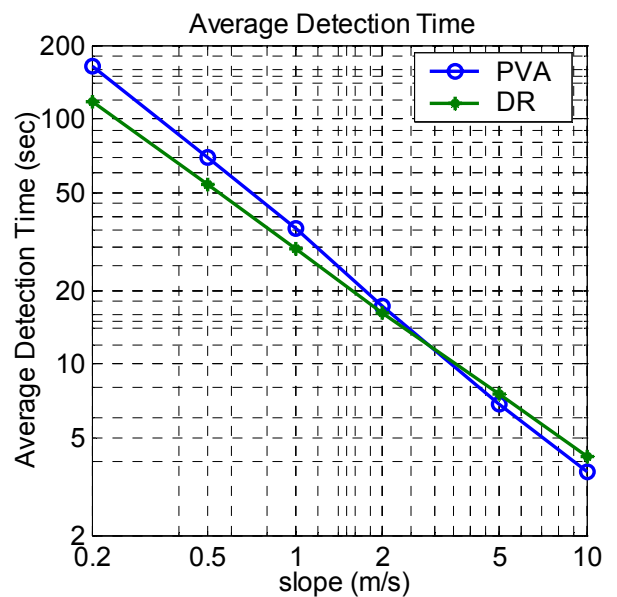

Fig. 2: ADT for ramp-type failure

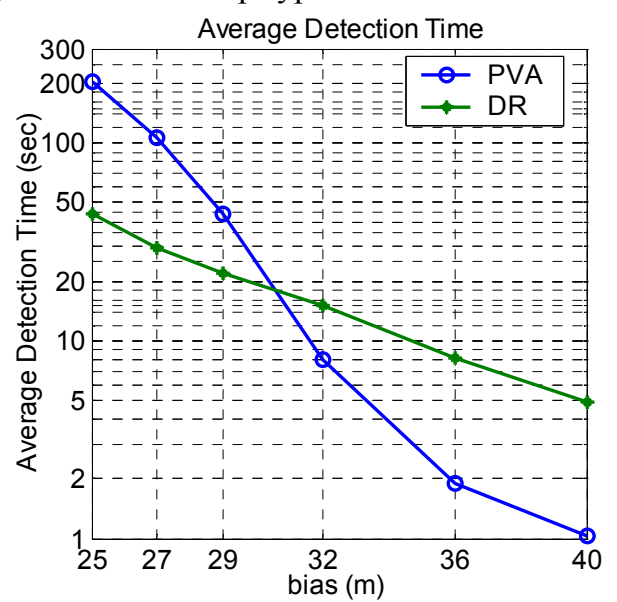

Fig. 3: ADT for step-type failure

In the first simulation a total of $1152(24 \times 48)$ space-time sample points are produced according to the user locations and simulation times (RTCA/DO208, 1991). The user locations covering the 24 geographic locations (RTCA/DO-208, 1991), and the simulation time is every half hour for 24 hours starting at midnight at the beginning of the GPS week. To simulate the satellite malfunction, both ramp-type failure and step-type failure are applied. The ramptype failure refers to a failure growing linearly with time, and the step-type failure refers to a constant bias happening at and remaining after a specified time. To detect the failed satellite, a detection time (DT) can be obtained for each point. DT is defined as the time needed from the onset of the failure to the annunciation of an alarm signal. The average detection time
(ADT) is the sample mean of the 1152 values of the DT. The simulation result of ADT for ramp-type pseudorange failure is plotted in Fig. 2. This figure shows that the ADT obtained through the DR equation is shorter than that through the PVA model under small ramp-type failures (slope $=0.2,0.5,1,2 \mathrm{~m} / \mathrm{s}$ ), i.e., the detection by using DR is faster. However, under large ramp-type failures (slope $=5,10 \mathrm{~m} / \mathrm{s}$ ), the $\mathrm{ADT}$ through the DR equation is longer than that through the PVA model, i.e., the detection by using PVA is faster. The simulation result of ADT for step-type pseudorange failure is plotted in Fig. 3. This figure shows that, the ADT through the DR equation is also shorter than that through the PVA model under small step-type failures (step $=20,27,29 \mathrm{~m}$ ). However, under large step-type failures (step $=32,36,40$ $\mathrm{m})$, the ADT through the DR equation is longer than that through PVA model.

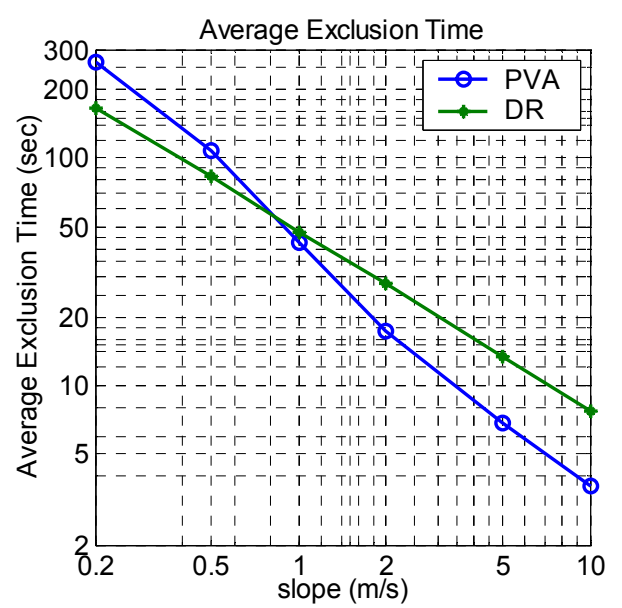

Fig. 4: AET for ramp-type failure

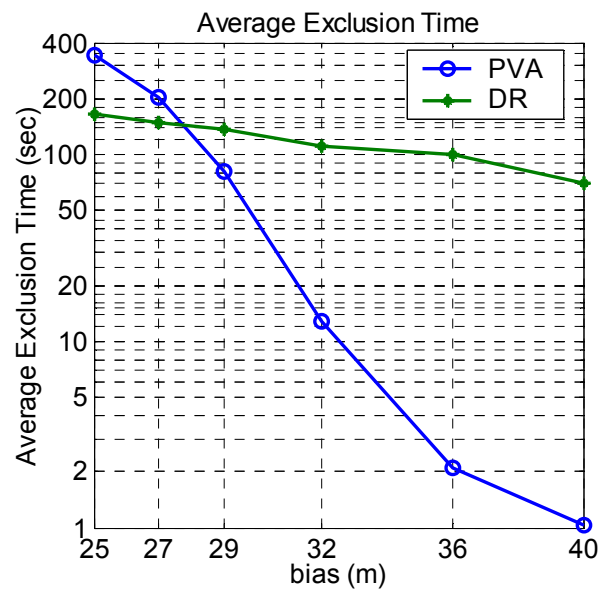

Fig. 5: AET for step-type failure

To exclude the failed satellite, an exclusion time (ET) can be obtained for each point. ET is defined as the time needed from the onset of the failure to the annunciation of a failure identification signal, and an ET can be obtained for each point. The average exclusion time (AET) is the sample mean of the 1152 values of the ET. The simulation result of AET for ramp-type pseudorange failure is plotted in Fig. 4. This figure shows that, under small ramp-type failures (slope $=0.2,0.5 \mathrm{~m} / \mathrm{s}$ ), the resulting AET of the DR equation is shorter than that of the PVA model, i.e., the exclusion by using DR is faster. While under large ramp-type failures (slope $=1,2,5,10 \mathrm{~m} / \mathrm{s}$ ), the resulting AET of the DR equation is longer than that of the PVA model, i.e., the exclusion by using PVA is 
faster. The simulation result of AET for step-type pseudorange failure is plotted in Fig. 5. This figure shows that, under small step-type failures (step $=25$, $27 \mathrm{~m}$ ), the resulting AET of the DR equation is shorter than that of the PVA model. While under large step-type failures $($ step $=29,32,36,40 \mathrm{~m})$, the resulting AET of the DR equation is longer than that of the PVA model.
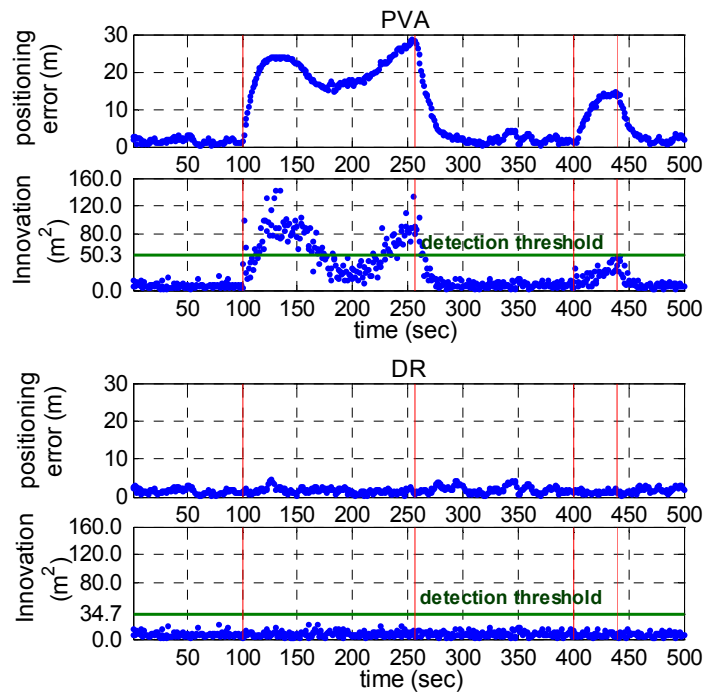

Fig. 6: Positioning errors and innovations

In the second simulation, both algorithms, PVA and DR, are applied to a vehicle which followed a constant course with a constant velocity $250 \mathrm{~m} / \mathrm{s}$ along the $\mathrm{x}$-axis until time $k=100$ seconds, at which point it began to maneuver in uniform circular motion with a radius of 12.5 kilometers and a centripetal acceleration of $5 \mathrm{~m} / \mathrm{s}^{2}$ until time $k=257.0796$ seconds, at which point it completed a half circle. Then the vehicle followed a constant course with a constant velocity $-250 \mathrm{~m} / \mathrm{s}$ along the $\mathrm{x}$-axis until time $k=400 \mathrm{~seconds}$, at which point it began to accelerate with a constant acceleration $-2.5 \mathrm{~m} / \mathrm{s}^{2}$ along the $\mathrm{z}$-axis until time $k=440$ seconds, at which point it began to course with a constant velocity $-250 \mathrm{~m} / \mathrm{s}$ along the $\mathrm{x}$-axis and $-100 \mathrm{~m} / \mathrm{s}$ along the $\mathrm{z}$-axis. The simulation results of applying the PVA and DR are plotted in Fig. 6. It shows that, in comparison with the PVA model, the proposed DR equation possess more accurate positioning results as a moving vehicle accelerates or slows down more rapidly, or as the vehicle corners at faster speeds. In addition, the normalized innovation squared (NIS) from the DR equation will still be chi-square distributed. Therefore, in comparison with the PVA model, the proposed DR can perform both positioning and FDE well as the vehicle manoeuvres.

\section{CONCLUSIONS}

Conventionally, the well known PVA model is adopted as the dynamic model of Kalman filter; however, the vehicle positioning result becomes less accurate as a moving vehicle accelerates or slows down more rapidly, or as the vehicle corners at faster speeds. Under this situation, the distribution of NIS is unknown, and thus is not suitable as the test statistic for FDE anymore. Therefore, the delta range equation is proposed by authors to accurately model the dynamic behavior of a maneuvering vehicle. Two simulations are conducted to verify the proposed al- gorithms. In the first simulation, Monte Carlo simulations are executed to compare the proposed DR equation with the conventional PVA model, where the vehicle is assumed to be moving with a constant velocity. In the case of step pseudorange failure, the simulation result of ADT might be too large to be acceptable. To overcome this problem, the algorithm based on the MA-filter (Tsai, et al., 2003) can be applied to reduce the ADT. In the second simulation, it is assumed that the vehicle will first complete a half circle at a specified time and then slows down rapidly at another time. Simulation results show that, in comparison with the PVA model, the proposed DR can provide more accurate positioning result and NIS. In summary, in comparison with the PVA model, the proposed DR can perform both positioning and FDE well as the vehicle manoeuvres.

\section{Reference}

Bar-Shalom, Y. and Fortman, T. E. (1993). Estimation and Tracking: Principles, Techniqus and Software, Artech House, Inc., Boston, London,

Brown, R. G. and Hwang, P. (1986). "GPS Failure Detection by Autonomous Means within the Cockpit," Proceedings of the Forty-Two Annual Meeting of the Institute of Navigation, June 24-26.

Da, R. and Lin, C.-F. (1995). “A New Failure Detection Approach and Its Application to GPS Autonomous Integrity Monitoring," IEEE transactions on Aerospace and Electronic Systems, Vol. 31, No. 1, pp. $499 \sim$ pp. 506

Fontana, D. R., Cheung, W., and Stansell T. (2001). "The Modernized L2 Civil Signal: Leaping Forward in the $21^{\text {st }}$ Century", GPS World, September, pp.28-34. (http://www.gpsworld.com)

Hofmann-Wellenhof, B., Lichtenegger, H, and Collins, J. (1997). Global Positioning System: Theory and Practice, (4th revised edition) Springer Wien New Work.

Parkinson, B. W. and Axelrad, P., (1988). "Autonomous GPS Integrity Monitoring Using the Pseudorange Residual", Navigation, Journal of the Institute of Navigation, Vol. 35, No.2, pp. 255-274.

Tsai, Y.-H., Chang, F.-R., and Yang, W.-C. (2004). "GPS Fault Detection and Exclusion Using Moving Average Filters," IEE Proceedings: Radar, Sonar and Navigation, Vol. 151, No. 4, pp 240-247.

Young, S. Y. R. and Mcgraw, G. A. (2003). "Fault Detection and Exclusion Using Normalized Solution Separation and Residual Monitoring Methods," Journal of the Institute of Navigation, Vol. 50, No. 3, pp151-169.

Document RTCA/DO-208 (1991). Minimum Operational Performance Standards for Airborne Supplemental Navigation Equipment Using Global Positioning System (GPS), Radio Technical Commission for Aeronautics.

Document RTCA/DO-229 (1997). Minimum Operational Performance Standards for Airborne Equipment Using Global Positioning System/Wide Area Augmentation System, Radio Technical Commission for Aeronautics. 